\title{
EDITORIAL
}

\section{The CARDia trial protocol}

\section{Smith}

The question of which revascularisation strategy is best in diabetics is a very important one, and it demands a robust answer

$A^{n}$ earlier edition of the web based version of Heart (May 2003) saw the electronic publication of the protocol of the CARDia (coronary artery revascularisation in diabetes) trial. The CARDia trial is a multi-centre, "prospective, randomised comparison of optimal coronary angioplasty-with use of stenting and abciximab recommended-versus up to date coronary artery bypass grafting in patients with diabetes mellitus suitable for either intervention". It will recruit 600 patients through 20 centres over a two year period. Patients suitable for either procedure will be randomised to percutaneous coronary intervention (PCI) or coronary artery bypass grafting (CABG). The PCI group will be further randomised to receive bare stents or rapamycin eluting stents.

The publication of a trial protocol is a new departure for Heart but follows precedents in other journals when important trials are planned or underway. It might at first sight seem odd to publish protocols of trials in peer review journals before the trial has completed and the results are available. After all a trial protocol without the results is little more than a well thought out idea and as such has little more value than reading the product of a bar-side brainstorming session among colleagues. Where will it end-will we see publication of lists of ideas that bubbled up at cardiologists' bath time? Clearly the gravity, relevance, and importance of a trial will determine whether the protocol should be published on its own. Does the CARDia trial meet such criteria and warrant web space? I believe it does.

At rates of $15-20 \%$, patients with diabetes form a very significant proportion of those undergoing revascularisation procedures and they are at considerably higher risk than non-diabetics

Table 1 Results of SoS (stent or surgery) study, showing diabetes has no effect on death or revascularisation rates

\begin{tabular}{|c|c|c|c|}
\hline & CABG & $\mathrm{PCl}$ & $\mathrm{p}$ Value \\
\hline \multicolumn{4}{|c|}{ Repeat revascularisation } \\
\hline DM & $4(5 \%)$ & $17(25 \%)$ & $p=0.54$ \\
\hline Non-DM & $26(6 \%)$ & $84(20 \%)$ & \\
\hline \multicolumn{4}{|c|}{ Death/myocardial infarction } \\
\hline DM & $9(12 \%)$ & $7(10 \%)$ & $p=0.57$ \\
\hline Non-DM & $40(9 \%)$ & $39(9 \%)$ & \\
\hline
\end{tabular}

$C A B G$, coronary artery bypass grafting; $D M$, diabetes mellitus; $\mathrm{PCl}$, percutaneous coronary intervention. whether they undergo percutaneous or surgical revascularisation. The five year survival for PCI in diabetics of $88 \%$ versus $93 \%$ in non-diabetics ${ }^{1}$ is mirrored in the surgical experience reported by Szabo and colleagues ${ }^{2}$ of $84.4 \%$ versus $91.3 \%$, where the increased postoperative morbidity is also highlighted. With such high prevalence and risk it behoves us to be absolutely clear about optimal management particularly with regard to revascularisation strategy. Yet current management strategies are based on generalisations supported by retrospective subgroup analyses of out of date trials. O'Neill made the much quoted statement that "Multivessel angioplasty in diabetics should be abandoned" ${ }^{\prime 3}$ after the publication of the diabetic subgroup analysis of the BARI trial ${ }^{4}$ revealed a significantly higher five year mortality in the PCI group. The trial, and possibly the statement, have had a significant effect on clinical practice. A brief review of the nine PCI versus CABG trials over the last 15 years shows how unlikely it is that such analyses have value. The proportion of diabetics recruited varies from $6 \%$ in the RITA trial ${ }^{5}$ to $27 \%$ in EAST $^{6}$ and in all but the very latest of these trials, the SoS trial, the diabetic subgroup was not prespecified. Of the multi-vessel balloon angioplasty versus CABG trials, the EAST trial showed no significant difference in mortality at five years compared to the $34.5 \%$ PCI versus $19.4 \%$ CABG $(p<0.003)$ reported in the BARI trial.

In the stent era the three trials ARTS, ERACI-2, and SoS show no significant difference in mortality between multi-vessel stenting and CABG in diabetics, but there the similarity between the trial results ends. In $\mathrm{ARTS}^{7}$ there is a significant increase in death in the diabetics undergoing PCI $(6.3 \%)$ compared with the non-diabetics $(1.6 \%, \mathrm{p}<0.011)$, although this is not reflected in the SoS results where there is no difference. Indeed in the SoS trial, the only trial with diabetes as a prespecified subgroup, the presence of diabetes has no effect on death or revascularisation rates at all (table 1$){ }^{8}$

Are we to believe the SoS trial because the diabetics were a prespecified subgroup? Fourteen per cent (142) of patients recruited were diabetic so the numbers are still relatively small. We are all aware that subgroup analysis should be viewed with great caution, but the erroneous effects are probably underestimated even in prespecified subgroups.

Abbreviations: ARTS, arterial revascularisation therapy study; BARI, bypass angioplasty revascularisation investigation; $C A B G$, coronary artery bypass grafting; CARDia, coronary artery revascularisation in diabetes EAST, Emory angioplasty versus surgery trial; $\mathrm{PCl}$, percutaneous coronary intervention; RITA, randomised intervention treatment of angina; SoS, stent or surgery 
One might be led to conclude from the trials so far that the advent of stents has removed any difference in mortality between PCI and CABG in diabetics with multivessel disease, but has left the problem of in-stent restenosis and consequently further revascularisations. Yet in the CARDia trial further revascularisation is only a major secondary end point, the primary end point being a composite of death/non-fatal myocardial infarction/non-fatal stroke. While it seems reasonable to have the end points in this order-after all the most important thing to know is which is the more dangerous procedure-the intriguing feature of this trial is whether the use of "optimal" PCI can not only produce lower morbidity but result in equivalent or less further revascularisation than "up to date" CABG.

\section{OPTIMAL PCI?}

The term "optimal PCI" is defined in the protocol as "the use of abciximab and a high rate of coronary stenting in conjunction with clopidogrel and aspirin". These features of PCI would be expected to make an impact on the early complications of PCI such as periprocedural infarction, no reflow, and subacute stent thrombosis, but not to have a particular effect on in-stent restenosis. According to the protocol the "aim of PCI is to achieve a less than $30 \%$ residual diameter stenosis together with normal TIMI III flow", but this seems remarkably unambitious and not optimal stenting. The angiographic target for optimal stenting of a lesion should surely be to leave no diameter stenosis since it is known from trials designed to investigate optimal stent deployment that even angiographic perfection will leave approximately $10 \%$ residual area stenosis when assessed by intravascular ultrasound. There are other things that one might introduce as adjuncts besides clopidogrel and abciximab to optimise the PCI. Troglitazone, for example, has been shown to reduce intimal hyperplasia in intracoronary stenting in diabetics at six months. ${ }^{10}$ The relatively low initial stenting target of less than 30\% residual stenosis might overemphasise the effects of the drug eluting stents in the secondary randomisation. The results of the Ravel ${ }^{11}$ trial are very encouraging with the same impressive effect on restenosis of rapamycin eluting stents apparently translating to the diabetics; but this is again unstratified, subgroup analysis and it will be very interesting to see the effects of drug eluting stents in formerly randomised diabetic patients. It is unfortunate to use the term optimal in a trial that will take some years to come to fruition since, like state of the art reviews that are rarely that by the time they get published, much can change.

\section{RISK STRATIFICATION}

One of the reasons for the variable results in the subgroup analyses of previous trials is the heterogeneity of diabetic populations. Not every diabetic has the same increased risk of mortality or in-stent restenosis after stent insertion and there are a number of factors known to affect the risk. For example the degree and duration of insulin resistance/diabetes predicts the degree of intimal hyperplasia. ${ }^{12}$ The interaction of advanced glycosylation end products with their vascular receptor, thought to be instrumental in restenosis, ${ }^{13}$ increases with age and duration of diabetes and renal failure. The risk, not only of restenosis but of mortality, is also shown to be related to the amount of proteinuria in diabetics. ${ }^{14}$ Without adequate risk stratification and appropriate randomisation of diabetic patients in revascularisation trials one would not expect to find consistent results. The authors of the CARDia trial have gone some way to stratify the diabetic patients according to both the duration of the diabetes and the treatment they are receiving — the latter might be viewed as a surrogate for severity of their insulin resistance-but there is no requirement to record the level of proteinuria which appears to be such an easy means of risk stratification. Is this an opportunity missed? In due course our ability to predict the patients at highest risk from death and restenosis will enable us to target them with specific tools designed to reduce the risk, but we must look for the markers and use the ones we find.

The question of which revascularisation strategy is best in diabetics is a very important one and demands a robust answer. The subgroup analyses of previous trials are confusing and a randomised controlled trial is required. The CARDia trial is a well thought out trial with realistic targets, the result of which should become a milestone in revascularisation in diabetes while the publication of its protocol is a little milestone in publishing for Heart.

\section{REFERENCES}

1 Stein B, Weintraub WS, Gebhart SP, et al. Influence of diabetes mellitus on early and late outcome after percutaneous transluminal coronary angioplasty. Circulation 1995;91:979-89.

2 Szabo Z Hakanson E, Svedieholm R, et al. Early postoperative outcome and medium-term survival in 540 diabetic and 2239 nondiabetic patients undergoing coronary artery bypass grafting. Ann Thorac Surg 2002; 74:712-9.

3 O'Neill W. Multivessel balloon angioplasty should be abandoned in diabetic patients. J Am Coll Cardiol 1998;31:20-2.

4 BARI Investigators. Influence of diabetes on 5 year mortality and morbidity in a randomised trial comparing CABG and PTCA inpatients with multivessel disease: the bypass angioplasty revascularisation investigation (BARI). Circulation 1997;96:1761-9.

5 RITA Trial Investigators. Coronary angioplasty versus coronary artery bypass surgery: the randomised intervention treatment of angina (RITA) trial. Lancet 1993;341:573-80.

6 King SB, III, Lembo NJ, Weintraub WS, et al. A randomised trial comparing coronary angioplasty with coronary artery bypass surgery. Emory angioplasty versus surgery trial (EAST). N Engl J Med 1994;331:1044-50.

7 Abizaid A, Costa MA, Centemero M, et al. Clinical and economic impact of diabetes mellitus on percutaneous and surgical treatment of multivessel coronary disease patients: insights from the arterial revascularisation therapy study (ARTS) trial. Circulation $2001 ; 104: 533-8$

8 Booth J, Stables RH, Clayton T, et al. Clinical outcome in diabetic patients with multi-vessel coronary artery disease treated with percutaneous coronary intervention compared to coronary bypass surgery: results from the stent or surgery (SoS) trial [abstract]. Heart (in press)

9 Brookes ST, Whitley E, Peters TJ, et al. Subgroup analyses in randomized controlled trial: quantifying the risks of false positives and false negatives. Health Technol Assess 2001;5(33): 1-56.

10 Takagi T, Akasaka T, Yamamuro A, et al. Troglitazone reduces neointimal proliferation after coronary stent implantation in patients with non insulin dependent diabetes mellitus: a serial intravascular ultrasound study. J Am Coll Cardiol 2000;36: 1529-35.

11 Morice MC, Serruys PW, Sousa JE, et al. A randomised comparisaon of a sirolimus-eluting stent with a standard stent for coronary revascularisation. N Engl J Med 2002;346:1773-801.

12 Pyorala $M$, Miettinen $H$, Laakso $M$, et al. Hyperinsulinaemia predicts coronary heart disease risk in healthy middle-aged men: the 22-year follow-up results of the Helsinki policemen study. Circulation 1998;98:398-404

13 Aronson D. Potential role of advanced glycosylation end products in promoting restenosis in diabetes and renal failure. Med Hypotheses 2002;59:297-301.

14 Marso SP, Ellis SG, Tuzcu M, et al. The importance of proteinuria as a determinant of mortality following percutaneous coronary revascularisation in diabetics. J Am Coll Cardiol 1999;33:1269-77.

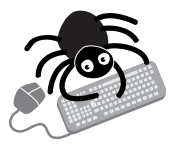

Visit the Heart website and access the supplemental article "The Coronary Artery Revascularisation in Diabetes (CARDia) trial: a prospective, randomised comparison of optimal coronary angioplasty with use of stenting and abciximab recommended versus up to date coronary artery bypass grafting in patients with diabetes mellitus suitable for either intervention", published in conjunction with the May 2003 issue - www.heartinl.com/supplemental 